\title{
High Molecular Weight (HMW): total adiponectin ratio is low in hiv-infected women receiving protease inhibitors
}

Fierdoz Omar $^{1 *}$, Joel A Dave ${ }^{2}$, Judy A King ${ }^{1}$, Naomi S Levitt ${ }^{2}$ and Tahir S Pillay ${ }^{1,3}$

\begin{abstract}
Background: At the time of the study, the HIV-treatment policy in South Africa included highly active antiretroviral therapy (HAART) regimens 1 (nucleotide reverse transcriptase inhibitors (NRTIs) only), and 2 (protease inhibitors (PI) and NRTIs). HAART is associated with the lipodystrophy syndrome, insulin resistance and reduced total adiponectin (TA) levels. The high molecular weight (HMW):TA ratio is a superior marker of insulin resistance. The aim of this study was to establish whether HMW:TA ratios are low in patients on PIs and whether they correlate with insulin resistance.
\end{abstract}

Methods: This was a cross-sectional study undertaken in an antiretroviral clinic at a tertiary hospital. The participants were 66 HIV-infected females: 22 were on regimen 2 (PI group), 22 on regimen 1 (non-PI) and 22 treatment naïve (TN), matched for BMI and age. Patients with a history of diabetes or impaired glucose tolerance were excluded. Serum adiponectin multimers were analysed using the AlpcoTM Adiponectin (Multimeric) enzyme immunoassay. Waist hip ratios (WHR), glucose and insulin levels were assessed, and HOMA-IR and QUICKI calculated. Data were analysed non-parametrically and multivariate analysis was performed.

Results: TA and HMW levels were lower in the treatment groups than in the TN group. HMW:TA was lower in the $\mathrm{PI}$ than in the non-PI and TN groups, and in the non-PI than in the TN groups. HMW:TA correlated negatively with waist, insulin and HOMA-IR, independently of BMI and duration of therapy. HOMA-IR and QUICKI did not differ among the groups.

Conclusion: HMW:TA is significantly decreased with HAART (particularly with PIs, but also with non-PIs) and may be a more sensitive marker of insulin resistance in these patients than conventional markers or HMW and total adiponectin individually.

Keywords: HMW adiponectin, Lipodystrophy syndrome, HMW: adiponectin ratio, Protease inhibitors, Insulin resistance

\section{Background}

Adiponectin is an insulin-sensitising hormone found in multimeric forms in the circulation with the high molecular weight $(\mathrm{HMW}) 16-18 \mathrm{mer}(>400 \mathrm{kDa})$ being the predominant and active form [1].

Although an adipokine, unlike other hormones secreted by adipocytes, adiponectin levels are reduced in people with increased central body fat [1], insulin resistance, type

\footnotetext{
* Correspondence: fierdoz.omar@nhls.ac.za

'Division of Chemical Pathology, C17 NHLS, Groote Schuur Hospital, University of Cape Town, Anzio Road Observatory, Cape Town 7925, South Africa

Full list of author information is available at the end of the article
}

2 diabetes mellitus and atherosclerosis, as well as in individuals with lipoatrophy and lipohypertrophy [1]. HMW adiponectin has been shown to correlate better with insulin sensitivity than total adiponectin (TA) [2] and the HMW:TA ratio to be a better predictor of coronary artery disease than TA [3]. The ratio has also been shown to be suppressed in type 2 diabetes mellitus patients with coronary artery disease even when HMW and TA levels were unchanged [4].

In HIV-associated lipodystrophy, a syndrome consisting of fat redistribution, dyslipidaemia and insulin resistance, adiponectin levels are significantly lower, demonstrating a 
negative correlation with abdominal visceral fat mass and insulin resistance $[5,6]$. This syndrome is associated with antiretroviral (ARV) therapy, particularly protease inhibitors (PIs) (but also nucleotide- and nucleoside reverse transcriptase inhibitors such as stavudine (d4T), zidovudine $(\mathrm{AZT})$ ) and didanosine (ddI) $[7,8]$. In such patients, thiazolidinedione administration, via peroxisome proliferatoractivated receptor $\gamma$ activation, leads to improved insulin sensitivity [9] with upregulation of adiponectin levels, specifically the HMW form $[10,11]$. Adiponectin administration in mice markedly ameliorates protease-induced dyslipidaemia, suggesting that hypoadiponectinaemia may be partially responsible for the metabolic derangements associated with PIs [12].

In South Africa, the National Department of Health had two highly active antiretroviral therapy (HAART) regimens at the time of this study. The first regimen consisted of $\mathrm{d} 4 \mathrm{~T}$, lamivudine (3TC) and either efavirenz (EFV) or nevirapine i.e. a combination of two nucleotide reverse transcriptase inhibitors (NRTIs) and one non-nucleotide reverse transcriptase inhibitor (NNRTIs); while the second regimen consisted of AZT and lopinavir/ritonavir (LPV/r). AZT and ddI are nucleotide- and nucleoside reverse transcriptase inhibitors, respectively, while $\mathrm{LPV} / \mathrm{r}$ is a PI.

The purpose of this study was to establish whether PI therapy was associated with lower HMW:TA ratios in HIV-infected patients, and to examine associated biochemical evidence of insulin resistance in these patients.

\section{Methods}

This cross-sectional study was performed in accordance with the Helsinki Declaration. The protocol was approved by the University of Cape Town Faculty of Health Sciences Research Ethics Committee with reference number REC 450/2006. Sixty-six HIV-infected African females were enrolled into the study into three groups, viz. PI (Regimen 2 for at least six months), non-PI (Regimen 1 for at least six months) and treatment naïve (TN) groups, each consisting of 22 patients. Subjects were recruited from the ARV clinic at Groote Schuur Hospital, with the non-PI and TN groups matched to the PI group for body mass index (BMI) and age. Exclusion criteria included a history of impaired glucose tolerance or diabetes mellitus, active acute opportunistic infections, renal failure and pregnancy. Written informed consent was obtained. Waist and hip circumferences, weight and height were measured, and the BMI and waist: hip ratio (WHR) calculated. A $75 \mathrm{~g}$ OGTT was performed and blood samples drawn at 0 and $120 \mathrm{~min}$. Glucose was measured at both time points and insulin and multimeric adiponectin in the 0 min sample only. Samples were centrifuged and stored at $-70^{\circ} \mathrm{C}$ until analysis. The homeostatic model assessment for insulin resistance (HOMA-IR) and quantitative insulin-sensitivity check index (QUICKI) were calculated.
Adiponectin was analysed using the Alpco $^{\mathrm{Tm}}$ Adiponectin (Multimeric) enzyme immunoassay (sensitivity $0.04 \mathrm{ng} /$ $\mathrm{mL}$ and coefficient of variation $(\mathrm{CV})<15 \%)$, insulin by the Bayer ACS180 auto-analyser (CV 12\%), and glucose by the Bayer Alera chemistry analyser.

\section{Statistical analysis}

Results were analysed non-parametrically, using the Mann-Whitney U, Kruskall-Wallis and Spearman correlation tests. Multivariate analyses and power calculations were performed. A p-value of 0.05 was considered significant.

\section{Results}

\section{Patient characteristics}

In the PI group, 21 patients received LPV/r and one Atazanavir for at least six months, with the median duration on PIs being 11.5 months. The median duration on regimen 1 drugs prior to progression to regimen 2 was 15 months. Four (18\%) patients received d4T and 13 (59\%) AZT, as part of their regimen. The median total duration on these drugs were 15.5 and nine months, respectively. In the non-PI group, 21 of the 22 patients received either AZT or d4T, with median durations of 12 and 10 months, respectively.

The median (interquartile range (IQR)) age and BMI among the groups were $36(29 ; 42)$ years and $27(24 ; 30)$, respectively. Waist measurement and WHR did not differ among the groups (Table 1).

\section{Adiponectin levels in serum (Figure 1 and Table 1)}

TA levels were within the reference interval (3.5 $22 \mathrm{mg} / \mathrm{L}$ ) [13] in all groups. However, total and HMW adiponectin levels were significantly lower in both treatment groups compared to the naïve group, with no significant difference between the PI and non-PI groups. In contrast, the HMW:TA ratio differed among all three groups and was significantly lower in the PI group than in both the non-PI and TN groups, and lower in the non-PI group than in the TN group. This difference was maintained when adjusting for BMI (data not shown) (Table 1 and Figure 1).

\section{Traditional markers of insulin resistance}

There were no significant differences among the groups for insulin, fasting and 2-h glucose, and the derived parameters HOMA-IR and QUICKI (Table 1). However, 15 (23\%) of the entire study sample of HIV-infected patients had insulin resistance, defined as HOMA-IR $>1.95$ [14], and their HMW:TA ratios were lower $(\mathrm{p}=0.0059)$ (median 0.31, IQR 0.22 and 0.47) compared to those without insulin resistance (median 0.50, IQR 0.40 and 0.57). Similarly, $40(60 \%)$ of the entire sample of HIVinfected patients were overweight $(\mathrm{BMI} \geq 25)$ and had 
Table 1 Adiponectin and markers of insulin resistance among the treatment groups (A), duration of therapy among the groups (B)

\begin{tabular}{|c|c|c|c|c|c|}
\hline \multicolumn{6}{|c|}{ (A) Adiponectin and markers of insulin resistance among the treatment groups } \\
\hline & PI Median (IQR) & Non-PI Median (IQR) & TN Median (IQR) & Units & p-value \\
\hline Age & $34.5(29.8 ; 41.0)$ & $34.5(31.3 ; 41.0)$ & $35.5(29.0 ; 41.0)$ & Years & 0.986 \\
\hline BMl & $27.7(24.9 ; 30.4)$ & $25.0(23.8 ; 29.5)$ & $25.7(22.9 ; 29.7)$ & - & 0.586 \\
\hline Waist & $89(82 ; 95)$ & $83(80 ; 93)$ & $82(76 ; 88)$ & $\mathrm{cm}$ & 0.194 \\
\hline WHR & $0.87(0.82 ; 0.93)$ & $0.85(0.79 ; 0.89)$ & $0.84(0.80 ; 0.90)$ & - & 0.315 \\
\hline Fasting insulin & $5.5(2.7 ; 10.1)$ & $3.1(2.1 ; 5.7)$ & $4.4(2.0 ; 8.0)$ & $\mathrm{mIU} / \mathrm{L}$ & 0.365 \\
\hline Fasting glucose & $86(81 ; 90)$ & $91(83 ; 98)$ & $91(85 ; 97)$ & $\mathrm{mg} / \mathrm{dL}$ & 0.145 \\
\hline 2 hour glucose & $104(80 ; 113)$ & $94(85 ; 109)$ & $94(81 ; 109)$ & $\mathrm{mg} / \mathrm{dL}$ & 0.877 \\
\hline HOMA-IR & $1.18(0.54 ; 2.09)$ & $0.72(0.50 ; 1.17)$ & $1.05(0.41 ; 1.73)$ & - & 0.479 \\
\hline QUICKI & $0.37(0.34 ; 0.42)$ & $0.41(0.37 ; 0.43)$ & $0.38(0.35 ; 0.45)$ & - & 0.234 \\
\hline Total adiponectin & $5.6(3.4 ; 9.4)$ & $7.3(4.0 ; 8.7)$ & $9.0(7.6 ; 12.1)$ & $\mathrm{ng} / \mathrm{mL}$ & $0.039^{*}$ \\
\hline HMW adiponectin & $2.2(1.0 ; 3.9)$ & $3.5(1.8 ; 5.1)$ & $5.3(3.8 ; 7.0)$ & $\mathrm{ng} / \mathrm{mL}$ & $0.002^{*}$ \\
\hline HMW:Total adiponectin & $0.35(0.29 ; 0.42)$ & $0.48(0.41 ; 0.56)$ & $0.56(0.52 ; 0.61)$ & - & $<0.0001^{* *}$ \\
\hline \multicolumn{6}{|c|}{ (B) Duration of therapy among the treatment groups } \\
\hline Regimen 1 & $15(8.5 ; 21.5)$ & $12(9.5 ; 20.0)$ & - & Months & \\
\hline Regimen 2 & $11.5(9.3 ; 14.0)$ & - & - & Months & \\
\hline$d 4 \mathrm{~T}$ & $15.5(9.8 ; 22.3)$ & $10(8 ; 17.5)$ & - & Months & \\
\hline $\mathrm{AZT}$ & $9(5 ; 16.5)$ & $12(11 ; 15.0)$ & - & Months & \\
\hline
\end{tabular}

The Kruskall-Wallis and Mann-Whitney $\mathrm{U}$ tests were used to assess differences among the groups collectively and individually, respectively. $\mathrm{PI}$, protease inhibitor group; Non-PI, non-protease inhibitor group; TN, treatment-negative group; IQR, interquartile range; HMW, high molecular weight. * $\mathrm{p}<0.05$; ${ }^{* *} \mathrm{p}<0.001$. Multiply by the following conversion factor for SI units: glucose 0.0555 (mmol/L); insulin 6.945 (pmol/L).

significantly lower $(\mathrm{p}=0.0475)$ HMW:TA ratios (median 0.44 , IQR 0.31 and 0.54 ) than those who were not overweight (median 0.50, IQR 0.44 and 0.58).

HMW, TA, and their ratio correlated negatively $(\mathrm{p}<0.05)$ with waist, BMI, fasting insulin and HOMA-IR, even when adjusted for BMI (Table 2). While HMW, TA, and their ratio correlated positively $(\mathrm{p}<0.05)$ with QUICKI, this significance was lost when adjusting for BMI. When adjusting for both BMI and total duration of therapy, only the HMW:TA ratio maintained a significant correlation with fasted insulin, HOMA-IR and QUICKI, while all three adiponectin parameters remained significantly associated with waist. TA, HMW and their ratio maintained their association with waist, fasting insulin and HOMA-IR when adjusting for duration of therapy alone. HMW and HMW:TA also remained significantly associated with QUICKI when adjusting for duration of therapy alone.

\section{Discussion}

Altered body composition and insulin resistance are components of the HIV-associated lipodystrophy syndrome [7]. This syndrome is primarily seen in HIV-infected patients on HAART, with a $17 \%$ risk of developing lipodystrophy after the first year of HAART and each additional 6 month period associated with a 45\% risk [15]. These findings are particularly associated with PIs, but similar findings have been seen in some patients on nucleotide and nucleoside analogues such as d4T, AZT and ddI [8].

Adiponectin has been implicated in the pathogenesis of HIV - associated lipodystrophy [12], with PI administration in mice producing a dose-related reduction in adiponectin levels and administration of recombinant adiponectin ameliorating the associated dyslipidaemia.

It was therefore somewhat surprising that TA levels were well within the reference interval in all our study groups. However, they were significantly lower in the HIVinfected patients on HAART therapy (both PIs and nonPIs) than in those not receiving treatment.

The active HMW form of adiponectin has been shown to correlate better with insulin sensitivity than TA [16], and has been shown to be low in HIV-infected patients with insulin resistance [10]. In our study, HMW adiponectin levels were significantly lower in patients receiving both PIs and non-PIs, but also in those receiving only non-PIs. This finding in the latter group, may be attributed to the use of $\mathrm{d} 4 \mathrm{~T}$ (16 (66\%) patients) or AZT (5 (23\%) patients), drugs which have both been associated with lipodystrophy [17].

The HMW:TA ratio is a superior marker of insulin resistance compared to total and HMW adiponectin levels individually [18]. It is also an independent risk factor for coronary vascular disease (CVD) [19]. Previous studies have shown increased cardiovascular risk in HIV-infected 
(a)

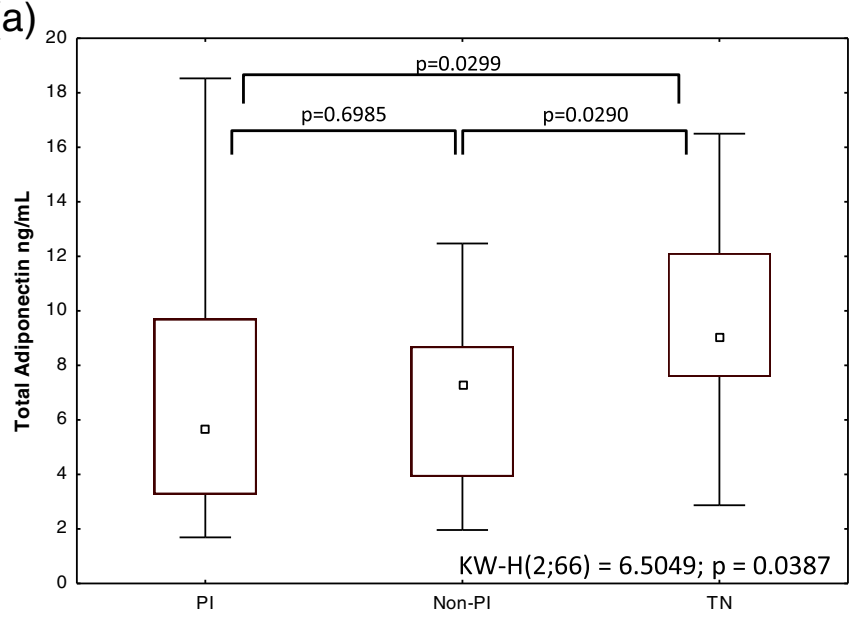

(b)

Group

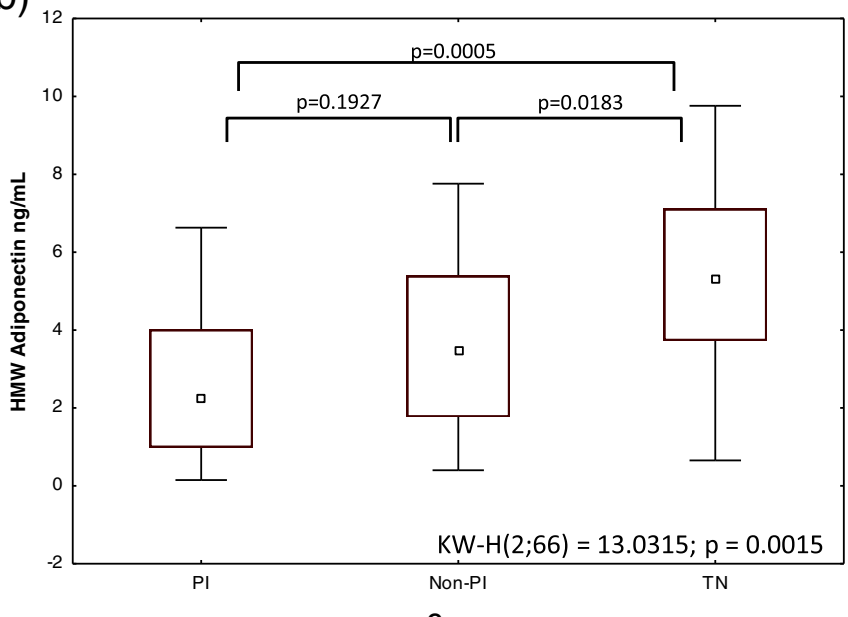

(c)

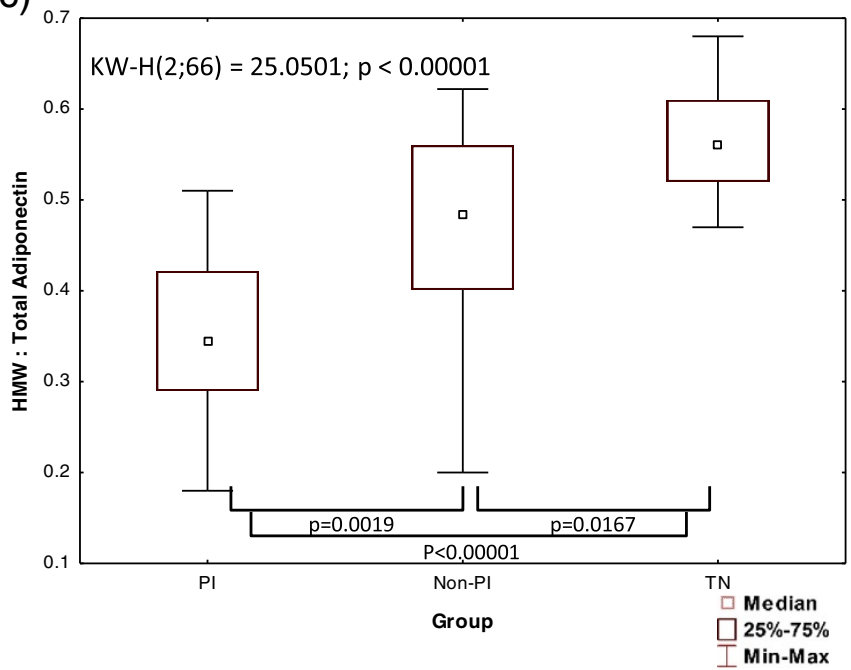

Figure 1 Distribution of (a) Total adiponectin, (b) HMW adiponectin, and (c) HMW: total adiponectin ratio among the three groups. HMW, high molecular weight; Pl, protease inhibitor group; Non-PI, non-protease inhibitor group; TN, treatment-negative group; KW, Kruskall-Wallis; $p<0.05$ considered significant. 
Table 2 The relationship between adiponectin and various markers of insulin resistance before and after adjusting for BMI and duration of therapy, separately and together

\begin{tabular}{|c|c|c|c|c|c|c|c|c|c|c|c|c|}
\hline \multicolumn{13}{|c|}{ Relationship between adiponectin and markers of insulin resistance } \\
\hline & \multirow[b]{3}{*}{ b } & \multicolumn{2}{|l|}{ Univariable } & \multicolumn{6}{|c|}{ Multivariable } & & & \\
\hline & & \multirow[b]{2}{*}{$95 \% \mathrm{Cl}$} & \multirow[b]{2}{*}{ p-value } & \multicolumn{3}{|c|}{ Adjusted for BMI } & \multicolumn{3}{|c|}{ Adjusted for duration of therapy } & \multicolumn{3}{|c|}{ Adjusted for BMI and duration of therapy } \\
\hline & & & & b & $95 \% \mathrm{Cl}$ & p-value & b & $95 \% \mathrm{Cl}$ & p-value & b & $95 \% \mathrm{Cl}$ & p-value \\
\hline \multicolumn{13}{|c|}{ Total Adiponectin } \\
\hline Waist & -0.27 & $(-0.3895,-0.1420)$ & $0.0001^{* *}$ & -0.28 & $(-0.5153,-0.0517)$ & $0.019^{*}$ & -0.29 & $(-0.4582,-0.1161)$ & $0.002^{*}$ & -0.35 & $(-0.6479,-0.0492)$ & $0.028^{*}$ \\
\hline Fasted Insulin & -0.28 & $(-0.4683,-0.0906)$ & $0.005^{*}$ & -0.21 & $(-0.3945,-0.0164)$ & $0.037^{*}$ & -0.28 & $(-0.5408,-0.0266)$ & $0.036^{*}$ & -0.24 & $(-0.4935,0.0099)$ & 0.067 \\
\hline HOMA-IR & -1.11 & $(-1.8677,-0.3428)$ & $0.006^{*}$ & -0.81 & $(-1.5741,-0.0551)$ & $0.040^{*}$ & -1.21 & $(-2.3196,-0.1036)$ & $0.038^{*}$ & -1.07 & $(-2.1435,0.0051)$ & 0.058 \\
\hline QUICKI & 14.20 & $(1.2607,27.1444)$ & $0.035^{*}$ & 8.24 & $(-4.8207,21.3036)$ & 0.221 & 15.25 & $(-0.2780,30.7806)$ & 0.061 & 11.29 & $(-4.4271,26.9992)$ & 0.167 \\
\hline \multicolumn{13}{|c|}{ HMW Adiponectin } \\
\hline Waist & -0.17 & $(-0.2484,-0.0923)$ & $0.0001^{* *}$ & -0.19 & $(-0.3350,-0.0429)$ & $0.014^{*}$ & -0.16 & $(-0.2637,-0.0627)$ & $0.003^{*}$ & -0.19 & $(-0.3674,-0.0109)$ & $0.044^{*}$ \\
\hline Fasted Insulin & -0.17 & $(-0.2941,-0.0547)$ & $0.006^{*}$ & -0.13 & $(-0.2475,-0.0078)$ & $0.041^{*}$ & -0.17 & $(-0.3204,-0.0169)$ & $0.035^{*}$ & -0.14 & $(-0.2918,0.0048)$ & 0.065 \\
\hline HOMA-IR & -0.69 & $(-1.1753,-0.2091)$ & $0.007^{*}$ & -0.51 & $(-0.9903,-0.0273)$ & $0.042^{*}$ & -0.72 & $(-1.3745,-0.0666)$ & $0.037^{*}$ & -0.64 & $(-1.2681,-0.0022)$ & 0.056 \\
\hline QUICKI & 9.38 & $(1.2388,17.5231)$ & $0.027^{*}$ & 5.67 & $(-2.5563,13.9009)$ & 0.182 & 9.97 & $(0.9112,19.0352)$ & $0.037^{*}$ & 7.66 & $(-1.5098,16.8289)$ & 0.110 \\
\hline \multicolumn{13}{|l|}{ HMW: TA } \\
\hline Waist & -0.007 & $(-0.0102,-0.0042)$ & $0.000^{* *}$ & -0.011 & $(-0.0165,-0.0054)$ & $0.0003^{* *}$ & -0.16 & $(-0.2637,-0.0627)$ & $0.003^{*}$ & -0.007 & $(-0.0130,-0.0016)$ & $0.016^{*}$ \\
\hline Fasted Insulin & -0.008 & $(-0.0123,-0.0030)$ & $0.002^{*}$ & -0.006 & $(-0.0109,-0.0014)$ & $0.013^{*}$ & -0.007 & $(-0.0119,-0.0025)$ & $0.005^{*}$ & -0.006 & $(-0.0111,-0.0018)$ & $0.009^{*}$ \\
\hline HOMA-IR & -0.03 & $(-0.0497,-0.0123)$ & $0.002^{*}$ & -0.03 & $(-0.0442,-0.0063)$ & $0.011^{*}$ & -0.03 & $(-0.0509,-0.0103)$ & $0.005^{*}$ & -0.03 & $(-0.0479,-0.0084)$ & $0.008^{*}$ \\
\hline QUICKI & 0.42 & $(0.1036,0.7331)$ & $0.011^{*}$ & 0.30 & $(-0.0230,0.6253)$ & 0.073 & 0.44 & $(0.1586,0.7148)$ & $0.004^{*}$ & 0.38 & $(0.0911,0.6598)$ & $0.014^{*}$ \\
\hline
\end{tabular}

HMW, high molecular weight; $b$, coefficient; $\mathrm{Cl}$, confidence interval. ${ }^{*} \mathrm{p}<0.05 ;{ }^{* *} \mathrm{p}<0.001$. 
patients, including those on HAART [20,21]. We found this ratio to be significantly lower in the PI and less so (but nevertheless still significantly so) in the non-PI groups. This may imply an increased risk for CVD in patients on HAART (consistent with the findings of others) [20,21], with the risk for CVD increased when both PIs and non-PIs are being used. This significantly lower HMW:TA ratio in patients receiving both PIs and NRTIs (with the majority, i.e. 17 of 22, of patients also receiving a thymidine analogue) confirms previous findings that protease inhibitors and thymidine analogues induce metabolic complications synergistically [7]. A limitation in this study is that the PI group also contained the nucleoside analogue ddI. Further studies are needed in the absence of so called d-drugs to confirm the contribution of PI drugs to the changes seen in this study.

Our patient groups were selected to exclude the presence of overt diabetes, and therefore it was not surprising that fasting glucose was not abnormal in any of the groups. On the other hand, we were surprised by the lack of difference in all measures of glucose tolerance and insulin sensitivity among the groups (Table 1), despite the difference seen among the groups in total and HMW adiponectin. However, a larger study sample may be required to verify this. The sample size was sufficiently powered (75.5\%) to detect a difference in HMW: TA, but not to verify the lack of significant differences demonstrated for the insulin resistance markers. A further limitation is that visceral and subcutaneous adiposity were not quantitatively assessed. Notwithstanding, the relationship between insulin resistance markers and adiponectin, previously shown [5], was demonstrated here, with the HMW:TA ratio shown to be significantly lower in patients (in all groups) with established insulin resistance (HOMA-IR greater than 1.95 [21]) and also significantly lower in patients with BMI greater than or equal to 25 than in those with lower BMIs. Furthermore, adiponectin levels (high, total and their ratio) correlated negatively with HOMA-IR and positively with QUICKI. The HMW:TA ratio also correlated negatively with waist, waist hip ratio (data not shown), fasting insulin levels and BMI (data not shown) all markers of insulin resistance. While BMI and duration of drug therapy collectively contribute to the association seen between the markers of insulin resistance and the TA and HMW adiponectin forms, they do not affect the associations seen with the HMW:TA ratio.

\section{Conclusion}

These data demonstrate that both PI- and non-PIcontaining HAART regimens significantly lower the HMW:TA ratio in HIV patients, with the ratio more significantly decreased in the PI-containing regimen, implying that PIs and NRTIs have an additive effect on the HMW:TA ratio. Although the HMW:TA ratio correlated negatively with indirect markers of insulin resistance, insulin resistance was not demonstrated to be associated with ARV drugs. We therefore propose that the HMW:TA ratio may be an earlier or more sensitive marker of insulin resistance in HIV-infected patients on HAART than conventional markers or HMW and total adiponectin individually.

\section{Abbreviations}

HMW: High molecular weight; HIV: Human immunodeficiency virus; PI: Protease inhibitor; d4T: Stavudine; AZT: Zidovudine; HAART: Highly active antiretroviral therapy; 3TC: Lamivudine; EFV: Efavirenz; ddl: Didanosine; NRTI: nucleotide reverse transcriptase inhibitors; NNRTI: non-nucleotide reverse transcriptase inhibitor; LPV/r: Liponavir/ritonavir; TA: Total adiponectin; ARV: Antiretroviral; non-PI: non-protease inhibitor; BMI: Body mass index; TN: Treatment naive; WHR: Waist: hip ratio; HOMA-IR: Homeostatic model assessment for insulin resistance; QUICKI: Quantitative insulin-sensitivity check index; CV: Coefficient of variation; IQR: interquartile range; CVD: coronary vascular disease; KW: Kruskall-Wallis.

\section{Competing interests}

The authors declare that they have no competing interests.

\section{Authors' contributions}

FO: carried out the analysis, produced the data, analysed the data and produced the draft of the manuscript. JD, DL: physicians, under whose care the patients fell; obtained the blood samples. JAK: assisted with obtaining the data and the laboratory analysis; TSP: conceptualised the study, designed the study, obtained the funding and supervised the research. All authors read and approved the final manuscript.

\section{Authors' information}

FO: Chemical Pathologist; JD, Consultant Endocrinologist ; NL, Professor of Diabetes and Endocrinology; TSP: Professor and Chair, Chemical Pathology and Clinical Pathology.

\section{Acknowledgements}

The authors wish to acknowledge Dr Andrew Boulle for assistance with statistical analysis. This project was funded by the National Health Laboratory Service, National Research Foundation of South Africa, World Diabetes Foundation and the Department of Health. This work was submitted as part fulfilment for the MMed degree in Chemical Pathology at the University of Cape Town.

This paper was supported by a grant from the National Health Laboratory Service Research Trust Fund, the National Research Foundation, the World Diabetes Foundation and the Department of Health.

\section{Author details}

'Division of Chemical Pathology, C17 NHLS, Groote Schuur Hospital, University of Cape Town, Anzio Road Observatory, Cape Town 7925, South Africa. ${ }^{2}$ Division of Diabetic Medicine and Endocrinology, Groote Schuur Hospital and University of Cape Town, Cape Town, South Africa. ${ }^{3}$ Department of Chemical Pathology, University of Pretoria and NHLS Tshwane Academic Division/Steve Biko Academic Hospital, Tshwane, South Africa.

Received: 2 July 2014 Accepted: 30 October 2014 Published: 16 December 2014

\section{References}

1. Swarbrick MM, Havel PJ: Physiological, pharmacological, and nutritional regulation of circulating adiponectin concentrations in humans. Metab Syndr Relat Disord 2008, 6(2):87-102.

2. Fisher FM, Trujillo ME, Hanif W, Barnett AH, McTernan PG, Scherer PE, Kumar S: Serum high molecular weight complex of adiponectin correlates better with glucose tolerance than total serum adiponectin in indo-asian males. Diabetologia 2005, 48(6):1084-1087.

3. El-Menyar A, Rizk N, Al Nabti AD, Hassira SA, Singh R, Abdel Rahman MO, Suwaidi JA: Total and high molecular weight adiponectin in patients with coronary artery disease. J Cardiovasc Med (Hagerstown) 2009, 10(4):310-315. 
4. Aso Y, Yamamoto R, Wakabayashi S, Uchida T, Takayanagi K, Takebayashi K, Okuno T, Inoue T, Node K, Tobe T, Inukai T, Nakano Y: Comparison of serum high-molecular weight (HMW) adiponectin with total adiponectin concentrations in type 2 diabetic patients with coronary artery disease using a novel enzyme-linked immunosorbent assay to detect HMW adiponectin. Diabetes 2006, 55(7):1954-1960.

5. Addy CL, Gavrila A, Tsiodras S, Brodovicz K, Karchmer AW, Mantzoros CS: Hypoadiponectinemia is associated with insulin resistance, hypertriglyceridemia, and fat redistribution in human immunodeficiency virus-infected patients treated with highly active antiretroviral therapy. J Clin Endocrinol Metab 2003, 88(2):627-636.

6. Tong Q, Sankale JL, Hadigan CM, Tan G, Rosenberg ES, Kanki PJ, Grinspoon SK, Hotamisligil GS: Regulation of adiponectin in human immunodeficiency virus-infected patients: Relationship to body composition and metabolic indices. J Clin Endocrinol Metab 2003, 88(4):1559-1564.

7. Carr A, Samaras K, Burton S, Law M, Freund J, Chisholm DJ, Cooper DA: A syndrome of peripheral lipodystrophy, hyperlipidaemia and insulin resistance in patients receiving HIV protease inhibitors. AIDS 1998, 12(7):F51-8.

8. Haubrich RH, Riddler SA, DiRienzo AG, Komarow L, Powderly WG, Klingman K, Garren KW, Butcher DL, Rooney JF, Haas DW, Mellors JW, Havlir DV, AIDS Clinical Trials Group (ACTG) A5142 Study Team: Metabolic outcomes in a randomized trial of nucleoside, nonnucleoside and protease inhibitorsparing regimens for initial HIV treatment. AIDS 2009, 23(9):1109-1118.

9. Sutinen J, Kannisto K, Korsheninnikova E, Fisher RM, Ehrenborg E, Nyman T, Virkamaki A, Funahashi T, Matsuzawa Y, Vidal H, Hamsten A, Yki-Jarvinen H: Effects of rosiglitazone on gene expression in subcutaneous adipose tissue in highly active antiretroviral therapy-associated lipodystrophy. Am J Physiol Endocrinol Metab 2004, 286(6):E941-9.

10. Qurashi S, Mynarcik DC, McNurlan MA, Ahn H, Ferris R, Gelato MC: Importance of the high-molecular-mass isoform of adiponectin in improved insulin sensitivity with rosiglitazone treatment in HIV disease. Clin Sci (Lond) 2008, 115(6):197-202.

11. Blumer RM, van der Valk M, Ackermans M, Endert E, Serlie MJ, Reiss P, Sauerwein HP: A rosiglitazone-induced increase in adiponectin does not improve glucose metabolism in HIV-infected patients with overt lipoatrophy. Am J Physiol Endocrinol Metab 2009, 297(5):E1097-104.

12. Xu A, Yin S, Wong L, Chan KW, Lam KS: Adiponectin ameliorates dyslipidemia induced by the human immunodeficiency virus protease inhibitor ritonavir in mice. Endocrinology 2004, 145(2):487-494.

13. Nien JK, Mazaki-Tovi S, Romero R, Erez O, Kusanovic JP, Gotsch F, Pineles BL, Gomez R, Edwin S, Mazor M, Espinoza J, Yoon BH, Hassan SS: Plasma adiponectin concentrations in non-pregnant, normal and overweight pregnant women. J Perinat Med 2007, 35(6):522-531.

14. Jennings $C L$, Lambert EV, Collins M, Joffe Y, Levitt NS, Goedecke JH: Determinants of insulin-resistant phenotypes in normal-weight and obese black african women. Obesity (Silver Spring) 2008, 16(7):1602-1609.

15. Martinez E, Mocroft A, Garcia-Viejo MA, Perez-Cuevas JB, Blanco JL, Mallolas J, Bianchi L, Conget I, Blanch J, Phillips A, Gatell JM: Risk of lipodystrophy in HIV-1-infected patients treated with protease inhibitors: A prospective cohort study. Lancet 2001, 357(9256):592-598.

16. Seino $Y$, Hirose $H$, Saito I, Itoh $H$ : High molecular weight multimer form of adiponectin as a useful marker to evaluate insulin resistance and metabolic syndrome in japanese men. Metabolism 2007, 56(11):1493-1499.

17. Bogner JR, Vielhauer V, Beckmann RA, Michl G, Wille L, Salzberger B, Goebel FD: Stavudine versus zidovudine and the development of lipodystrophy. J Acquir Immune Defic Syndr 2001, 27(3):237-244.

18. Hara K, Horikoshi M, Yamauchi T, Yago H, Miyazaki O, Ebinuma H, Imai Y, Nagai R, Kadowaki T: Measurement of the high-molecular weight form of adiponectin in plasma is useful for the prediction of insulin resistance and metabolic syndrome. Diabetes Care 2006, 29(6):1357-1362.

19. Wang W, Xing W, Zhang H, Ding M, Shang L, Lau WB, Wang X, Li R: Reduced high molecular weight adiponectin is an independent risk factor of cardiovascular lesions in hypercholesterolemic patients. Clin Endocrinol (Oxf) 2012.

20. Dolan SE, Hadigan C, Killilea KM, Sullivan MP, Hemphill L, Lees RS, Schoenfeld D, Grinspoon S: Increased cardiovascular disease risk indices in HIV-infected women. J Acquir Immune Defic Syndr 2005, 39(1):44-54.

21. Friis-Moller N, Sabin CA, Weber R, d'Arminio Monforte A, El-Sadr WM, Reiss P, Thiebaut R, Morfeldt L, De Wit S, Pradier C, Calvo G, Law MG, Kirk O, Phillips AN, Lundgren JD, Data Collection on Adverse Events of Anti-HIV Drugs (DAD) Study Group: Combination antiretroviral therapy and the risk of myocardial infarction. N Engl J Med 2003, 349(21):1993-2003.

doi:10.1186/1472-6890-14-46

Cite this article as: Omar et al:: High Molecular Weight (HMW): total adiponectin ratio is low in hiv-infected women receiving protease inhibitors. BMC Clinical Pathology 2014 14:46.

\section{Submit your next manuscript to BioMed Central and take full advantage of:}

- Convenient online submission

- Thorough peer review

- No space constraints or color figure charges

- Immediate publication on acceptance

- Inclusion in PubMed, CAS, Scopus and Google Scholar

- Research which is freely available for redistribution 